\title{
Aa. Vv., Actes du IIème Colloque International sur la Littérature en Moyen Français (Milan, 8-10 mai 2000)
}

\section{Antonella Amatuzzi}

\section{(2) OpenEdition}

1 Journals

\section{Edizione digitale}

URL: https://journals.openedition.org/studifrancesi/40362

DOI: $10.4000 /$ studifrancesi.40362

ISSN: 2421-5856

Editore

Rosenberg \& Sellier

\section{Edizione cartacea}

Data di pubblicazione: 1 juillet 2004

Paginazione: 161-163

ISSN: 0039-2944

\section{Notizia bibliografica digitale}

Antonella Amatuzzi, «Aa. Vv., Actes du llème Colloque International sur la Littérature en Moyen Français (Milan, 8-10 mai 2000)», Studi Francesi [Online], 142 (XLVIII | I) | 2004, online dal 30 novembre 2015, consultato il 09 septembre 2021. URL: http://journals.openedition.org/studifrancesi/40362 ; DOI: https://doi.org/10.4000/studifrancesi.40362

Questo documento è stato generato automaticamente il 9 septembre 2021.

\section{(c) 9 (i) $\Theta$}

Studi Francesi è distribuita con Licenza Creative Commons Attribuzione - Non commerciale - Non opere derivate 4.0 Internazionale. 


\title{
Aa. Vv., Actes du Ilème Colloque International sur la Littérature en Moyen Français (Milan, 8-10 mai 2000)
}

\author{
Antonella Amatuzzi
}

\section{NOTIZIA}

«L'analisi linguistica e letteraria», 1-2, anno VIII, 2000, Actes du IIème Colloque International sur la Littérature en Moyen Français (Milan, 8-10 mai 2000), réunis par SERGIO CIGADA, ANNA SLERCA, GIOVANNA BELLATI, MONICA BARSI, Milano, Vita e Pensiero, 2000, pp. 484.

1 Questo volume è articolato in tre sezioni: la prima ha come tema principale la poesia lirica (10 comunicazioni), la seconda François Villon (7 comunicazioni) mentre la terza riunisce studi su soggetti diversi (10 comunicazioni).

2 Marc-René JUNG, La naissance de la ballade dans la première moitié du XIVe siècle, de Jean Acart à Jean de le Mote et à Guillaume de Machaut, pp. 7-29. La critica ha sempre considerato che le prime ballate non cantate fossero apparse dopo Guillaume de Machaut. L'A., attraverso l'analisi di alcune ballate anteriori (quelle di Jean Acart del 1332, quelle di Jean de le Mote del 1339-1340) intende dimostrare che la svolta avvenne ben prima, con delle nuove sperimentazioni nella struttura metrica e nelle rime.

3 Marie-Françoise NOTZ, Grandeur et décadence du refrain du XIV au XVI siècle: sur quelques exemples de poèmes lyriques d'oc et d'oil, pp. 31-38. Nel Duecento e nel Trecento il «refrain» è caratteristico dei generi «popolareggianti» per il suo tipo di enunciazione che funziona come «memoria» del racconto. Nel Quattrocento il «refrain» perde la sua importanza quando si impone il sonetto venuto dall'Italia nel quale, tuttavia, il «beau vers» conclusivo può essere considerato un'eco del «refrain» medievale.

4 Roman REISINGER, Les allégories du jeu dans la poésie lyrique en moyen français, pp. 39-48. Nei testi letterari in «moyen français» il gioco ha una funzione di «exemplum» moraleggiante. Grazie all'allegoria il gioco è trasformato nella visione utopica di una 
vita armoniosa, soddisfacente e rilassata, in contrasto con le reali condizioni di vita, estremamente difficili.

James LAIDLAW, Les «Cent balades d'amant et de dame» de Christine de Pizan, pp. 49-63. L'A. si sofferma sul testo, conservato unicamente nel ms. Londra, British Library, Harley 4431, di cui Christine stessa avrebbe curato la presentazione e la decorazione e che alcuni critici definiscono autografo. Risalenti al 1406, queste «balades» costituiscono per Christine «les adieux à l'amour courtois» (p. 63).

6 Gianni mombello, Quatre poèmes à la Vierge de Giovan Giorgio Alione, pp. 65-106. L'A. offre l'opportunità di conoscere quattro poesie di Alione dedicate alla Vergine, di cui fornisce i testi in appendice. Ne descrive la metrica e il contenuto tematico: il primo sembra essere, almeno in parte, un «noël»; gli altri degli adattamenti di preghiere latine, rispettivamente "Obsecro te, Domina sancta Maria», «Salve Regina» e "Ave Maris Stella». Segue uno studio approfondito dell'ortografia, della morfologia, della sintassi e del lessico di questo poeta astigiano che scrive un francese eccellente.

7 Claudio GALDERISI, Visions mythiques du temps et 'remythisation du temps' dans le «Passe Temps» de Michault Taillevent et Pierre Chastellain, pp. 107-120. Si interroga sul modo in cui i poeti della fine del Medio Evo affrontano la questione del tempo, soffermandosi in particolare sul sistema dei tempi verbali nel Passe Temps di Michault de Taillevent e nel Temps perdu di Pierre Chastellain, esempi perfetti della «remythisation» del tempo, cioè «le pouvoir qu'a seul la réalité fictionnelle, artistique, d'évoquer et faire coïncider vision mythique du temps et actualisation de remythisation» (p. 114). Entrambi sono costruiti su una sequenza di presenti che creano un flusso temporale che mette in collisione il tempo interiore col tempo cosmico.

8 Giovanna Bellati, L'immortalité par l'art chez les poètes de la Grande Rhétorique, pp. 121-138. Il tema dell'immortalità dell'arte è un asse tematico portante della poetica del Cinquecento ed è già presente nell'opera di alcuni Grands Rhétoriqueurs in special modo nelle «complaintes» funebri, negli epitaffi e nelle «déplorations» rivolte ad artisti. L'A. ne ravvisa un'evoluzione: da semplice allusione a sviluppo importante, da celebrazione dell'artista a celebrazione dell'arte stessa che avvicina a Dio.

Adrian ARMSTRONG, La «Plainte du Désiré» de Jean Lemaire de Belges: du manuscrit illustré aux marges de l'imprimé, pp. 139-156. Mette in luce alcune varianti riscontrabili tra le versioni manoscritte e le edizioni a stampa della Plainte du Désiré, dominata, a livello tematico da due discorsi: uno commemorativo (in onore del defunto conte di Ligny) e uno estetico. Nei mss il discorso estetico è subordinato alla commemorazione mentre nelle edizioni a stampa l'attività creatrice assume maggiore importanza.

10 Anna Maria Finoli, 'Versi d'amore e prose di romanzi', pp. 157-175. Prende in esame alcuni romanzi del periodo compreso tra la seconda metà del Trecento e la seconda metà del Quattrocento, contenenti inserimenti lirici. Ne constata un'evoluzione cronologica: le inserzioni vere e proprie si fanno sempre di più rare e il lirismo sopravvive attraverso passaggi in prosa di registro lirico.

11 Jean BALSAMO, Les collections des anciens poètes français de Galliot du Pré (1528-1533), pp. 177-194. Indaga la ricezione della poesia in «moyen français» nel Cinquecento attraverso la «politica editoriale» del libraio parigino Galliot du Pré. Nel pubblicare i testi degli antichi poeti egli procedette ad un vero e proprio esercizio di filologia applicando ai testi recenti il lavoro riservato ai classici latini dando loro, oltre che «une plus value savante, une réelle valeur ajoutée marchande» (p.191). 
12 Sergio CIGADA, L'anacoluthe comme figure stylistique et la structure du «Testament de François Villon», pp. 197-207. Prende in esame la prima strofa e la strofa iniziale della seconda parte (versi 793-800) del Testament, accomunate dalla presenza della figura grammaticale dell'anacoluto. Secondo l'A., attraverso questa figura stilistica che rappresenta «la marque de la liberté» (p. 206), Villon indica che nella sua opera si mescoleranno i soggetti più vari in assenza di ogni regola e sottolinea la sua libertà di spirito. In riferimento alla struttura del Testament i due anacoluti attestano l'intenzione di Villon di dividere il poema in due parti, secondo due linee direttrici precise: liricomorale e satirico-sociale.

Rika VAN DEYCK, Le discours d'identification dans le «Testament» de Villon, pp. 209-227. Si interessa all'uso che Villon fa della funzione attributiva quando parla di se stesso per concludere che, per mezzo dei qualificanti e delle relazioni tra espressioni che determinano l'identità dell'autore, Villon vuole raggiungere lo scopo di ottenere giustizia e perdono delle sue colpe attraverso la parola poetica.

14 Anna SLERCA, La structure thématique du "Testament» de François Villon, pp. 229-244. Propone una suddivisione della struttura del Testament sulla base di criteri tematici: una prima parte (versi 1-832) affronterebbe il problema della Fortuna, il secondo (versi 833-1265) quello della giustizia, il terzo (versi 1266-2023) quello della pedagogia e dell'educazione.

15 Jelle кoopmans, Denise et la Tante Laurence (Villon «Testament» v. 1234 et «Pathelin» v. 148), pp. 245-255. Denise e Tante Laurence, citate rispettivamente nel Testament e nella Farce possono essere, secondo le interpretazioni, dei nomi propri di donne veramente esistite o invece indicare dei tipi e costituire dunque delle vere e proprie locuzioni. Senza fornire una risposta definitiva l'A. sottolinea come una tale questione faccia riflettere sul sistema ermeneutico della locuzione in «moyen français».

16 Giuseppe Antonio BRUNELLI, 'Tant grate chevre que mal gist...' La ballade de Villon dite des Proverbes (sagesse populaire et autobiographie), pp. 257-267. Fornisce una lettura della ballata intesa come racconto autobiografico del poeta il quale, attraverso i proverbi, riesce a tradurre la saggezza e l'esperienza umana (e la sua in particolare) in poesia.

17 Jean DUfOURNeT, Villon et Paris ou le grand Carnaval, pp. 269-280. Villon si presenta come poeta di Parigi e della città. Parigi è, nello spirito del poeta, uno spazio chiuso e protetto ma diventa anche uno spazio interiore. È un luogo caotico, in perenne mutamento, carnevalesco, ambiguo, «force de vie et source de mort» che il poeta interiorizza e sul quale si proietta.

Patrizio TUCCI, Villon contre Franc Gontier, pp. 281-302. Intende reinserire la ballata di Villon in «risposta» al Dit de Franc Gontier di Philippe de Vitry nella scia dei testi in cui si tratta della campagna e dei suoi abitanti. Situando in un contesto urbano il locus amoenus di tutte le possibilità di felicità stravolge le prospettive precedenti in modo sovversivo.

19 Earl Jeffrey RICHARDS, Le concept de Droiture chez Christine de Pizan et sa pensée politique, pp. 305-314. All'inizio del Livre de la Cité des Dames appaiono tre figure allegoriche, Raison, Droiture et Justice. Quella che risulta più innovativa è Droiture. Secondo l'A. «elle semble représenter pour Christine une allégorie expérimentale correspondante au concept juridique d'aequitas» (p. 310) che le consente di proporre una giustizia rinnovata dalla ragione e quindi una nuova libertà per le donne della Cité. 
20 Anne schoysman, Les prologues de Jean Miélot, pp. 315-328. Si concentra sui prologhi che accompagnano le traduzioni di Miélot nelle quali non si trovano dichiarazioni teoriche o metodologiche. Egli si presenta come «translateur» e non come «compilateur» e cerca piuttosto «l'homogénéité dans les rapports entre iconographie, texte et prologues» (p. 328).

21 Maria colombo timelli, Le «Cligès» en prose (1455), ou l'actualisation d'un ancien 'conte' en vers, pp. 329-342. Ripercorre la fortuna critica dell'adattamento in prosa del Cligès del 1455, ne presenta i tratti salienti soffermandosi sui cambiamenti che subisce il personaggio di Artus, sull'interesse che certi dettagli potevano destare nella corte di Philippe le Bon e sull'organizzazione del testo, di cui annuncia l'edizione critica.

Pierre DEMAROLLE, Antoine de La Sale et le texte, pp. 343-353. Predilige una lettura dell'opera di Antoine de La Sale che concepisce il testo come un insieme di idee e di fatti non sottomessi esplicitamente ad un sistema concettuale e logico. Osserva così che per il «compilateur» La Sale la concatenazione e lo sviluppo di blocchi di testo non ha meno importanza della redazione stessa.

G. Matteo Roccati, La «Moralité de Fortune, Maleur, Eur, Povreté, Franc Arbitre et Destinée». Une adaptation $d u$ "Certamen paupertatis et fortune» (Boccace, "De Casibus», livre III), pp. 355-382. Si occupa di un adattamento teatrale del Certamen paupertatis et fortune, estratto dal terzo libro del De casibus di Boccaccio, una moralità anonima e inedita di più di 2400 versi. Ne sottolinea i tratti salienti e, attraverso un'analisi comparativa con testi similari, ne stabilisce la datazione intorno agli anni 1450-1475.

Paola CIFARELLI, Le manuscrit BNF fr. 15.219: quelques remarques à propos des extraits de la traduction du «De casibus» de Boccace, pp. 383-397. Dopo una descrizione del ms. in questione, l'A. riflette sulla funzione che riveste, all'interno del codice, la seconda versione francese, ad opera di Laurent de Premierfait, del Paupertatis et fortune certamen boccaccesco. Fornisce poi delle considerazioni di ordine stilistico e codicologico sul testo tradotto e, dopo averlo comparato con un altro codice che lo contiene, appartenuto a Eugénie Droz, ipotizza una diffusione isolata di questo capitolo del De casibus.

Valérie JouËT, Que deviennent les discours français dans la «Chronique du Religieux de SaintDenis»?, pp. 399-416. Studia le trasformazioni subite da due discorsi pronunciati in francese, il Vivat rex di Jean Gerson e l'Accipe sanctum gladium di Philippe de Villette, nel loro passaggio nella Chronique redatta in latino da Michel Pintoin. Il lavoro di traduzione e abbreviazione rispetta le caratteristiche principali del discorso.

Martin GOSMAN, Le pouvoir royal et l'idée de la 'nation' dans les comptes rendus de la réunion des États à Plessis-lès-Tours (1506), pp. 417-436. Prende in esame tre documenti che offrono interpretazioni della riunione degli Stati tenutasi nel 1506, nella quale fu questione di annullare il progetto di matrimonio della figlia unica del re, claude de France, con Carlo d'Asburgo (il futuro Carlo V) e di indicare invece Francesco d'Angoulême come futuro sposo e quindi erede al trono. Quello che stupisce è che in nessuno dei documenti si faccia menzione, come argomento in favore del «voltafaccia», della legge salica o di altri testi giuridici che impedivano che uno straniero salisse sul trono di Francia.

Monica BARSI, Jean d'Auton, poète et historiographe sous Louis XII, pp. 437-458. Illustra la personalità di Jean d'Auton, «chroniqueur» operante alla corte di Anne de Bretagne e Luigi XII, di cui fu storiografo, e la sua opera, circoscrivendola all'interno della 
produzione storiografica di fine Quattrocento e inizio Cinquecento, che si interroga sulla funzione della storia. Due sono le correnti principali: gli scrittori di attualità ai quali appartiene Jean d'Auton, e coloro che si occupano di grandi compilazioni.

Jennifer BRITNELL, L'Épître héroïque à la cour de Louis XII et d'Anne de Bretagne: le manuscrit fr. F.v.XIV.8 de Saint-Pétersbourg, pp. 459-484. Analizza il ms. fr F.v.XIV.8 della Biblioteca Nazionale di San Pietroburgo, contenente la traduzione in versi francesi delle Héroïdes di Ovidio, e la miniature che la accompagnano per stabilire l'influenza e la diffusione dell'épître, la sua trasformazione poetica a corte. Il genere è privato di tutto ciò che è passione, tragico ed anche elegiaco e sono invece sottolineati gli aspetti di ingeniosità per elogiare il vincitore. 